\title{
A dor de perder um filho no período perinatal: uma revisão integrativa da literatura sobre o luto materno
}

\section{The pain of losing a child in the perinatal period: an integrative literature review on maternal mourning}

\author{
Beatriz Gonçalves Lopes \\ Universidade Estadual de Ponta Grossa - UEPG - Ponta Grossa - Brasil \\ bia loppes@hotmail.com \\ Alessandra Rodrigues Martins \\ Universidade Estadual de Ponta Grossa - UEPG - Ponta Grossa - Brasil \\ aleeh martins@hotmail.com \\ Márcia Regina Carletto \\ Universidade Estadual de Ponta Grossa - UEPG - Ponta Grossa - Brasil \\ marciahcarletto@uol.com.br \\ Pollyanna Kássia de Oliveira Borges \\ Universidade Estadual de Ponta Grossa - UEPG - Ponta Grossa - Brasil \\ pollyannakassia@hotmail.com
}

\begin{abstract}
Resumo
O objetivo deste estudo foi identificar, em periódicos nacionais e internacionais, a produção bibliográfica relativa ao luto vivenciado por mães, e/ou familiares, que experimentaram a perda perinatal, no intuito de compreender as angústias, medos e as estratégias empregadas no apoio aos envolvidos, por meio de revisão integrativa da literatura publicados entre 2013 e 2017. Realizou-se busca bibliográfica em quatro bases de dados: Pubmed (U.S. National Library of Medicine); Bireme (Biblioteca Virtual em Saúde); Scopus e Scielo (Scientific Electronic Library Online), com descritores em ambas as bases, em português, inglês e espanhol. Verificou-se a baixa existência de estudos com evidência científica elevada sobre o luto materno e uma escassez sobre o tema na literatura brasileira. Os tipos de estudo mais empregados foram a pesquisa qualitativa e pesquisa bibliográfica, com nove artigos publicados em cada categoria de pesquisa. Porém, dentro dos 26 estudos revistos, encontrou-se também cinco estudos transversais, um estudo longitudinal, um metodológico e um caso-controle. Por meio deste estudo percebeu-se que as mães que vivenciam a perda de um filho no período perinatal sentem uma dor devastadora e que elas se apegam na fé para transpor este período e os sentimentos por ele provocados. Profissionais da saúde devem ser qualificados para assistência e apoio aos atores envolvidos nesse momento angustiante, favorecendo a possiblidade de rituais de despedida do bebê pela mãe e familiares, e retirando a mãe enlutada do convívio hospitalar de outras mães. Mas, há vários estudos qualitativos que mostram as subjetividades ligadas à dor da perda de um bebê.
\end{abstract}

Palavras-chave: luto, morte perinatal, literatura de revisão como assunto. 


\begin{abstract}
The aim of this study was to identify, in national and international journals, the bibliographic production related to grief experienced by mothers and / or family members who experienced perinatal loss, in order to understand the anguish, fears and strategies employed to support those involved, through an integrative literature review published between 2013 and 2017. A bibliographic search was performed in four databases: Pubmed (US National Library of Medicine); Bireme (Virtual Health Library); Scopus and Scielo (Scientific Electronic Library Online), with descriptors in both bases, in Portuguese and English. There was a low existence of studies with high scientific evidence on maternal mourning and a scarcity on the subject in the Brazilian literature. The most used types of study were qualitative research and bibliographic research, with 9 articles published in each research category. However, within the 26 reviewed studies, there were also 5 cross-sectional studies, 1 longitudinal study, 1 methodological study and 1 casecontrol. Through this study it was possible to realize that mothers who experience the loss of a child in the perinatal period feel a devastating pain and that they cling to the faith to overcome this period and the feelings caused by it. Health professionals should be qualified to assist and support the actors involved in this distressing moment, favoring the possibility of farewell rituals for the mother and family, and removing the bereaved mother from the hospital life of other mothers. But there are several qualitative studies that show the subjectivities linked to the pain of losing a baby.
\end{abstract}

Keywords: bereavement, perinatal death, review literature as topic.

\title{
1. Introdução
}

O óbito de um feto ou neonato contesta o curso vital esperado pela sociedade para uma criança. Quando acontece, os familiares podem passar por uma angústia intensificada, choque, raiva e desespero, e até mesmo um período longo de sofrimento (FLENADY et al., 2014; HILL et al., 2017).

Em geral, as mortes fetais e neonatais ocorrem de forma inesperada, e podem disparar problemas físicos e psicológicos nas mães que vivenciam este evento. Tristeza profunda, sentimentos depressivos ou de culpa, autoestima baixa, raiva, fracasso, vergonha, pensamentos suicidas são alguns sinais que a literatura relata estarem relacionados ao luto materno. Para a mãe, não se perde apenas o filho, mas sua identidade e uma parte de si (HILL et al., 2017). Essa situação pode levar ao isolamento do convívio social (STURROCK; LOUW, 2013; BOYDEN et al., 2015).

Os óbitos perinatais são aqueles que ocorrem a partir da $22^{a}$ semana gestacional até $06^{\circ}$ dia de vida da criança. Por serem óbitos de fetos ou recém-nascidos, muitas vezes são ignorados em relação ao sofrimento gerado aos familiares, em especial nas mães. Socialmente parece que o sofrimento materno deveria ser diretamente proporcional ao período de convivência com o filho. Assim, a dor da perda de um feto ou de um recémnascido muitas vezes é subestimada, ocultada e inexiste espaço social e cultural para sentir e expressar os sinais do luto. No entanto, muitas questões sobre o luto materno vêm sendo discutidas na atualidade, abrindo possibilidades para melhor compreensão e identificação de lacunas no conhecimento (CASELLATO, 2015).

Partindo do interesse em identificar o que a literatura recente apresentava sobre o luto de mães que perderam bebês no período perinatal este manuscrito teve por objetivo identificar, em periódicos nacionais e internacionais, a produção bibliográfica relativa ao luto vivenciado por mães, e/ou familiares, que experimentaram a perda perinatal, no intuito de compreender as angústias, medos, materno e as estratégias empregadas no apoio aos envolvidos. 


\section{Materiais e método}

Trata-se de uma revisão integrativa da literatura, com vistas a ampliar o aprendizado do tema, baseando-se em estudos anteriores e seguindo um método sistemático para obtenção dos resultados da pesquisa. Essa pesquisa seguiu o modelo proposto por Souza, Silva e Carvalho (2010) que descrevem as seis fases do processo de elaboração de uma revisão integrativa: $1^{\underline{a}}$ fase-elaboração da pergunta norteadora; $2^{\underline{a}}$ fase-busca ou amostragem na literatura; $3^{\underline{a}}$ fase-coleta de dados; $4^{\mathrm{a}}$ fase-análise crítica; $5^{\underline{a}}$ fase-discussão dos resultados; $6^{\underline{a}}$ fase-apresentação da revisão integrativa.

Uma vez definido o tema, elaborou-se a seguinte questão norteadora: qual o conhecimento científico, dos últimos 5 anos, produzido acerca do luto vivenciado por mães, e/ou familiares, que experimentaram a perda perinatal? Realizou-se a busca bibliográfica em quatro bases de dados: Pubmed (U.S. National Library of Medicine); Bireme (Biblioteca Virtual em Saúde); Scopus e Scielo (Scientific Electronic Library Online), com descritores em ambas as bases, em português e inglês. Os termos utilizados em língua inglesa foram: stillbirth AND grief OR mourning AND perinatal death $O R$ perinatal loss OR fetal death AND mother-child relations OR maternal-fetal relations. Em português, empregou-se: natimorto $A N D$ luto $A N D$ morte perinatal $O R$ mortalidade perinatal $O R$ morte fetal $A N D$ relação mãe-filho $O R$ relação mãe-feto.

A busca realizou-se em julho de 2017. Foram incluídos artigos nacionais e internacionais nas línguas inglesa, portuguesa e espanhola, sendo restrito ao período de publicação dos estudos para os últimos 5 anos (2013-2017). Procedeu-se a leitura dos títulos dos artigos encontrados para sua inclusão ou exclusão. Foram lidos os resumos ou abstracts dos textos que tiveram títulos ligados ao tema, a fim de confirmar a relação da pesquisa com esse. Para aqueles condizentes com o tema proposto, realizou-se a leitura completa do artigo e posterior utilização nessa revisão integrativa. Foram excluídos artigos que não especificaram o luto materno como tema principal.

Ao total, 274 publicações foram encontradas, estando distribuídas da seguinte forma: 254 no PubMed, 15 Bireme, três na Scielo e duas na Scopus. O processo de seleção dos artigos está resumido no fluxograma da Figura 1. Excluídas as repetições entre as bases de dados e os artigos não condizentes com o tema em questão, foram obtidas ao final 26 estudos utilizadas por esta revisão integrativa. Após a fase de leitura foi construído o Quadro $1 \mathrm{com}$ a produção encontrada, contendo os itens: autores, amostra, tipo de estudo, objetivo, principais achados e o nível de evidência, totalizando assim seis variáveis analisadas 
Figura 1: Fluxograma da seleção de artigos para a revisão de literatura

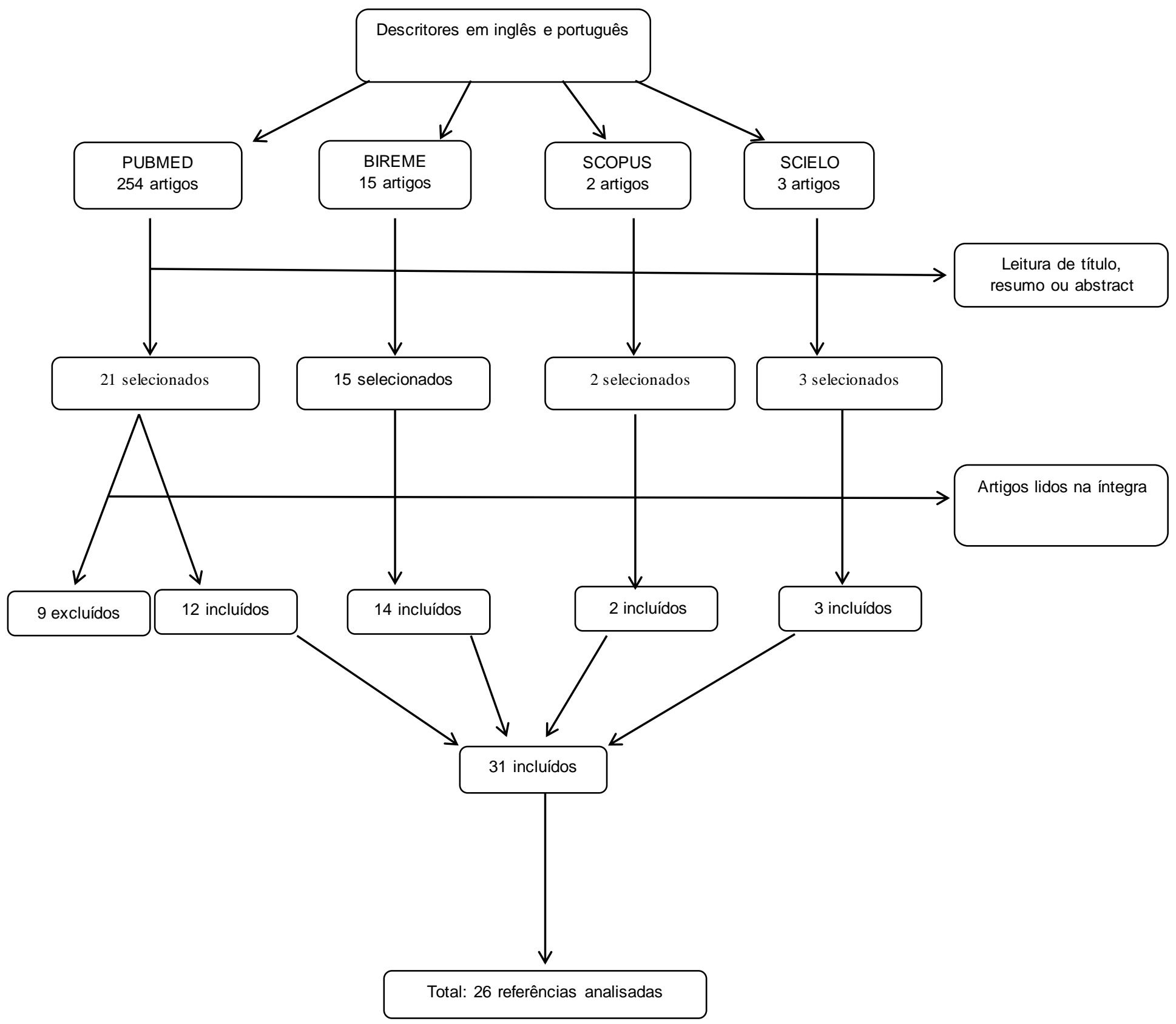

Fonte: Autoras (2018)

\section{Resultados}

Verificou-se que as publicações, na literatura brasileira, são escassas, encontrando apenas três artigos na base considerada. Já na literatura internacional, tem sido um tema bastante discutido. Entretanto, ainda demanda de mais pesquisas voltadas para a compreensão do luto materno e implantação de estratégias de apoio às mães enlutadas. No Quadro 1 são apresentados os achados das 26 publicações que fizeram parte desta revisão integrativa. 
Quadro 1: Autores, ano de publicação, amostra, tipo de estudo, objetivos e principais achados de estudos publicados entre 2013 e 2014 , referentes ao luto pela perda de uma criança no período perinatal.

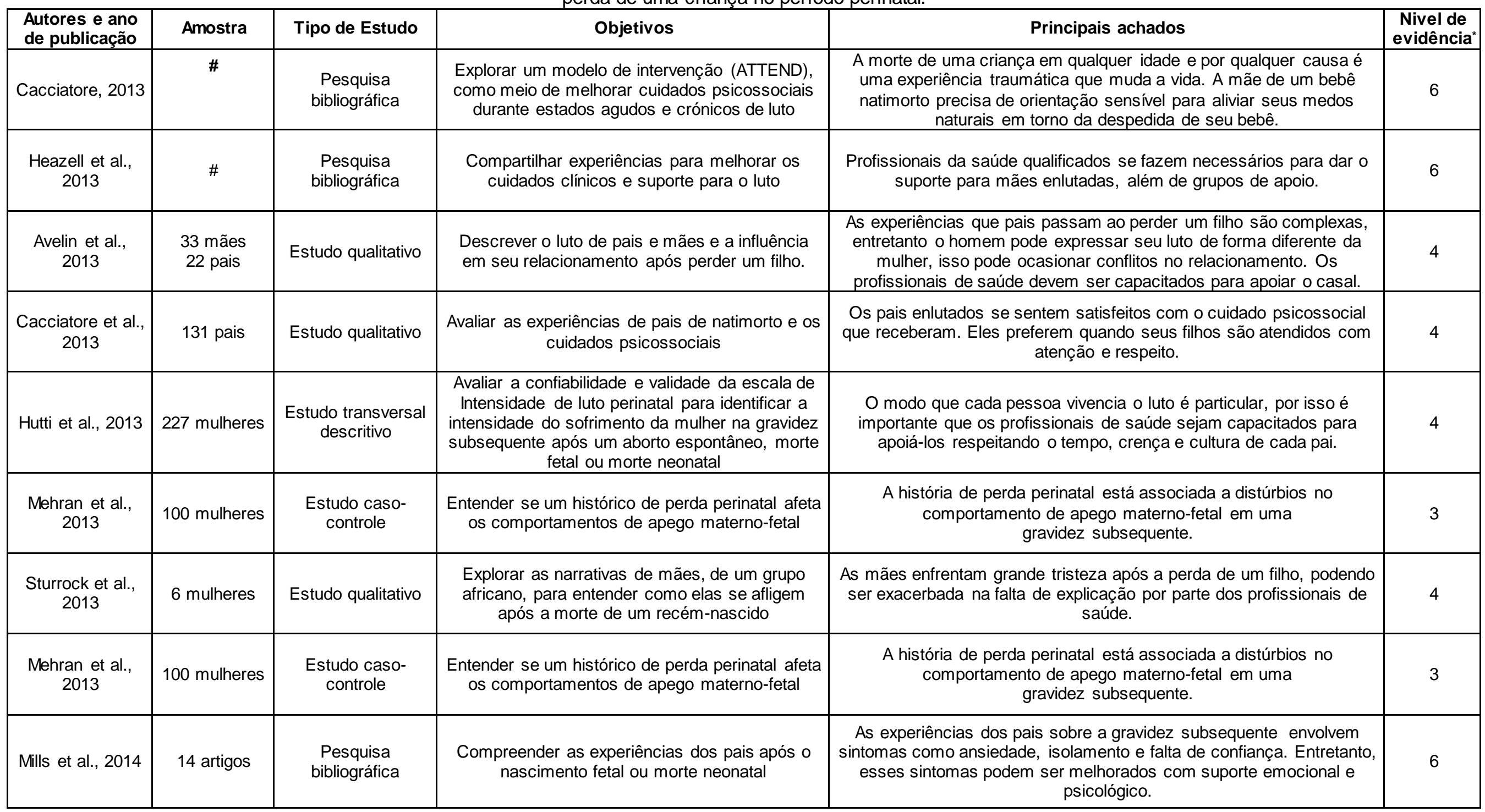




\begin{tabular}{|c|c|c|c|c|c|}
\hline $\begin{array}{l}\text { Murphy et al., } \\
2014\end{array}$ & $\#$ & $\begin{array}{l}\text { Pesquisa } \\
\text { bibliográfica }\end{array}$ & $\begin{array}{l}\text { Compreender a experiência dos avós que } \\
\text { perdem um neto no período perinatal }\end{array}$ & $\begin{array}{c}\text { Os avós esperam a vinda de um neto, e quando ocorre a morte } \\
\text { perinatal, esse momento é caracterizado pela tristeza da perda do } \\
\text { neto e da tristeza ao ver seu filho/filha sofrendo. Assunto que deve ser } \\
\text { mais discutido nas literaturas. }\end{array}$ & 6 \\
\hline $\begin{array}{l}\text { Campbell- } \\
\text { Jackson et al., } \\
2014\end{array}$ & 7 casais & Estudo qualitativo & $\begin{array}{l}\text { Compreender como pai e mãe vivenciam a } \\
\text { gravidez subsequente após uma perda fetal }\end{array}$ & $\begin{array}{c}\text { Pais apresentam sentimentos parecidos com as mães durante o } \\
\text { processo do luto, e ambos sentem ansiedade e medo na gravidez } \\
\text { subsequente. }\end{array}$ & 4 \\
\hline $\begin{array}{l}\text { Huberty et al., } \\
2014\end{array}$ & 24 mulheres & Estudo qualitativo & $\begin{array}{l}\text { Explorar as crenças das mulheres sobre a } \\
\text { atividade física após um nascimento fetal }\end{array}$ & $\begin{array}{l}\text { As mulheres sentem vergonha para praticar atividade física após seu } \\
\text { corpo estar modificado pela gravidez. As mães que relataram } \\
\text { praticarem atividade física falaram que esta ajudou a aliviar sintomas } \\
\text { depressivos e estavam se preparando para uma gravidez } \\
\text { subsequente. }\end{array}$ & 4 \\
\hline $\begin{array}{l}\text { Ryninks et al., } \\
2014\end{array}$ & 21 mulheres & Estudo qualitativo & $\begin{array}{l}\text { Investigar como as mães descrevem sua } \\
\text { experiência de passar o tempo com seu bebê } \\
\text { nascido e como elas se sentiram sobre a decisão } \\
\text { que eles fizeram para ver e segurar seu bebê }\end{array}$ & $\begin{array}{l}\text { A preparação antes do contato com o bebê, o suporte profissional } \\
\text { durante o contato, previnem problemas na saúde mental materna. }\end{array}$ & 4 \\
\hline Sun et al., 2014 & 151 hospitais & Estudo transversal & $\begin{array}{l}\text { Explorar relações de complicações de gravidez e } \\
\text { resultados dos nascimentos neonatais de uma } \\
\text { população }\end{array}$ & $\begin{array}{l}\text { As complicações na gravidez e morbidades perinatais estão } \\
\text { relacionadas como fatores de risco que afetam o resultado neonatal. }\end{array}$ & 3 \\
\hline
\end{tabular}

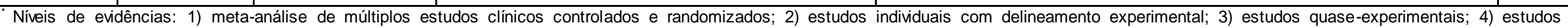
descritivos ou com abordagem qualitativa; 5) relatos de caso ou de experiência; 6) opiniões de especialistas (Souza; Silva; Carvalho, 2010).

Fonte: Autoras (2018)

Quadro 2: Autores, ano de publicação, amostra, tipo de estudo, objetivos e principais achados de estudos publicados entre 2015 e 2017 , referentes ao luto pela Autores e an

\begin{tabular}{|c|c|c|}
\hline $\begin{array}{c}\text { Autores e ano } \\
\text { de publicação }\end{array}$ & Amostra & Tipo de Estu do \\
\hline $\begin{array}{c}\text { Boyden et al., } \\
2015\end{array}$ & 12 estudos & $\begin{array}{c}\text { Pesquisa } \\
\text { bibliográfica }\end{array}$ \\
\hline $\begin{array}{c}\text { Peters et al., } \\
2015\end{array}$ & $\#$ & $\begin{array}{c}\text { Pesquisa } \\
\text { bibliográfica }\end{array}$ \\
\hline Petro, 2015 & $\#$ & $\begin{array}{c}\text { Pesquisa } \\
\text { bibliográfica }\end{array}$ \\
\hline
\end{tabular}
perda de uma criança no período perinatal.

\begin{tabular}{|c|c|c|}
\hline Objetivos & Principais achados & $\begin{array}{l}\text { Nivel de } \\
\text { evidência* }\end{array}$ \\
\hline $\begin{array}{l}\text { Descrever a dor e as implicações que pais afro- } \\
\text { americanos sofrem ao perder um filho }\end{array}$ & $\begin{array}{l}\text { O sofrimento dos pais causa sérias consequências para a saúde em } \\
\text { longo prazo. A consequência do luto dos pais afro-americanos é } \\
\text { semelhante ao de todos os pais, porém condicionado a fatores raciais, } \\
\text { discriminação e a desvantagem econômica. }\end{array}$ & 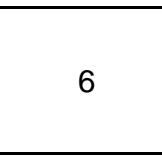 \\
\hline $\begin{array}{l}\text { Promover e informar uma prática baseada em } \\
\text { evidências, significativa e culturalmente } \\
\text { apropriada entre os prestadores de cuidados de } \\
\text { mães e famílias enlutadas }\end{array}$ & $\begin{array}{l}\text { Os pais querem que a equipe demonstre sensibilidade e empatia, } \\
\text { valide suas emoções e forneça informações claras. Além de apoiar e } \\
\text { orientar decisões sobre ver e segurar seu bebê. }\end{array}$ & 6 \\
\hline $\begin{array}{l}\text { Abordar as necessidades pastorais imediatas e } \\
\text { propor respostas de fé que os ministros podem } \\
\text { empregar para cuidar dos pais que sofrem da } \\
\text { perda perinatal }\end{array}$ & $\begin{array}{c}\text { É necessário treinamento e educação para cuidadores pastorais para } \\
\text { demonstrarem cuidados compassivos para pais enlutados e suas } \\
\text { famílias, e com a doação de seus dons de energia, determinação e } \\
\text { esperança. }\end{array}$ & 6 \\
\hline
\end{tabular}




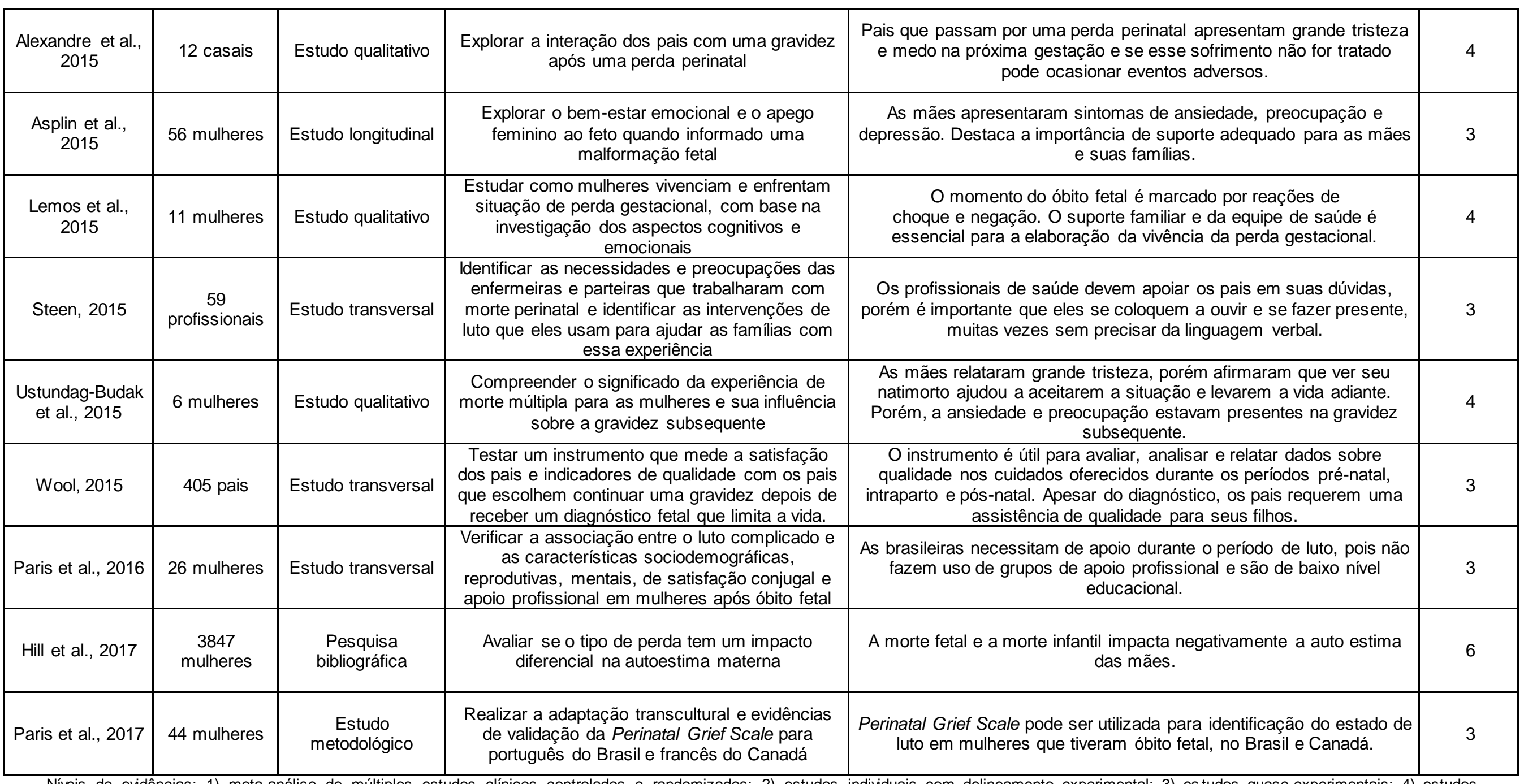

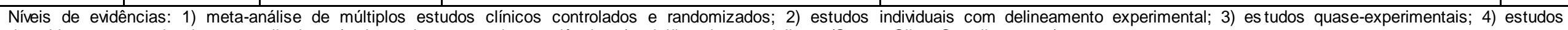
descritivos ou com abordagem qualitativa; 5) relatos de caso ou de experiência; 6) opiniões de especialistas (Souza; Silva; Carvalho, 2010).

Fonte: Autoras (2018) 
Notou-se que os tipos de estudos mais empregados foram a pesquisa qualitativa e bibliográfica, com nove artigos publicados em cada categoria de pesquisa. Porém, dentro dos 26 estudos revistos, encontrou-se também cinco estudos transversais, um longitudinal, um metodológico e um caso-controle. Apesar de a busca ter sido voltada para estudos que tratassem puramente do luto materno pós-perda perinatal, muitos estudos, além de avaliarem o luto materno, envolveram também os familiares, em especial os pais.

\section{Discussão}

Nesta revisão integrativa observou-se que os estudos qualitativos são os mais publicados sobre a temática da mortalidade perinatal e o luto a ela associado. A revisão aponta também que, dentre os estudos quantitativos, há poucos com maior nível de evidência. Em sua maioria apresentam nível de evidência 4 e 6, de acordo com o modelo de Souza, Silva e Carvalho (2010). Portanto, não foram encontradas publicações com meta-análises ou estudos experimentais, fato que enfatiza uma lacuna da literatura, e pode trazer consequências para o acompanhamento de mães e familiares enlutados, no sentido de desconhecer as ferramentas e terapêuticas mais efetivas que poderiam apoiar na reestruturação do ser materno após a perda da criança ou feto.

Por outro lado, como o tema do luto envolve os aspectos psíquicos e do sofrimento inerente à condição humana, os estudos qualitativos podem representar 0 maior quantitativo publicado porque a abordagem das entrevistas poderia favorecer o acesso aos enlutados. Além disso, conhecer o pensamento das pessoas apoiaria na tomada de decisões para além da abordagem numérica dos estudos quantitativos.

Campos, Onocko-Campos e Barrio (2013) afirmam que a partir do momento que o conceito de medicina baseada em evidências cresceu, fez com que a construção do conhecimento de forma indutiva se tornasse pouco científico, porém, a construção de dados, sejam estes quantitativos ou qualitativos é essencial, mas não suficiente para atribuir à uma verdade absoluta. Já existe uma vertente internacional onde sistemas de saúde utilizam tanto estudos qualitativos quanto quantitativos para basearem suas decisões, talvez sendo essa a melhor estratégia para a formulação de políticas públicas.

A tristeza ao vivenciar a perda de um filho foi um ponto comum encontrado entre todos os estudos. Acredita-se que os pais sofrem mais pela perda de um filho do que qualquer ser humano que passa pela dor da morte de um ente querido, isso se dá pela estreita relação entre pais e filhos. Essa dor não depende da idade da criança e nem do motivo, a dor é causada pela perda e muitas vezes não passa (BOYDEN et al., 2015).

Ao sofrerem uma perda perinatal, os pais têm maneiras divergentes de agir, alguns vivenciam o momento na hora e sentem grande tristeza, alguns podem ficar em choque por horas ou dias para então sentirem o luto (HUTTI; ARMSTRONG; MYERS, 2013).

Também, os autores concordam que as mães que perdem um filho demandam mais tempo no processo do luto. Algumas fases como negação, raiva, barganha, depressão e aceitação são visualizadas em todo o processo de luto materno. Entretanto, essas fases não têm uma ordem e tempo demarcado (LOPES et al., 2017).

O processo do luto é uma busca para integrar a morte do bebê de uma maneira que tenha um significado para a mãe e esta compreenda o momento que está vivendo. Entretanto, pais que não conseguem realizar essa ressignificação podem apresentar sofrimento prolongado (STURROCK; LOUW, 2013).

As mães enlutadas relatam vivenciar esse momento sozinhas, como se apenas elas fossem capazes de entender tamanha dor (LOPES et al., 2017). Alguma ajuda é ofertada, mas, nem sempre de forma apropriada. Frases como: "você é jovem" e "logo você terá outro" muitas vezes geram pressões nas mães podendo prolongar o processo do luto (PETRO, 2015). 
Um ponto levantado nos estudos é o fato de as mães enlutadas ficarem internadas na maternidade com mães não enlutadas. Por se tratar de um lugar com várias mães com seus bebês saudáveis, tal situação intensifica o sentimento de fracasso em relação à missão de ter um filho (LEMOS; CUNHA, 2015).

Um ponto importante para ajudar a família na reestruturação após a morte, e diminuir a dor materna, é a explicação dada aos pais sobre a morte do seu filho. Diante disso, a equipe de saúde deve explicar de forma clara aos pais o motivo do óbito (STURROCK; LOUW, 2013; FLENADY et al., 2014). Entretanto, muitos profissionais de saúde se sentem incapacitados para explicar, pela dor e revolta em que as mães estão vivendo (HEAZELL et al., 2013).

A falta de explicação pode fazer com que as mães se sintam culpadas do ocorrido, envergonhadas e isoladas de grupos sociais (CAMPBELL-JACKSON; BEZANCE; HORSCH 2014; PARIS; MONTIGNY; PELLOSO, 2017). Encontrar o significado da morte do seu filho traz benefícios para a vida dos pais e finaliza o processo do luto, porém esse caminho é doloroso e muitas vezes insuportável. Dessa maneira, é necessário que os profissionais da saúde, representantes religiosos, amigos e familiares estejam presentes, com disponibilidade para acolher as queixas, fazer uma escuta qualificada, aconselhar quando necessário, e dar o suporte que necessitem (PETRO, 2015).

Além do apoio, deve-se oportunizar um momento para as mães sanarem suas dúvidas. Além de encaminhá-las para serviços de psicologia e grupos terapêuticos com o objetivo de apoiar e ajudar nesse processo (CACCIATORE, 2013a). De outro modo, ao programar uma gravidez subsequente à perda de um filho, as mães encontram outras dificuldades comumente mencionadas pela literatura: podem se sentir inseguras e angustiadas, necessitando de ajuda de pessoas próximas, principalmente, seus companheiros (CAMPBELL-JACKSON; BEZANCE, HORSCH, 2014).

Casais que vivenciam a perda de um filho podem apresentar períodos intensos de sofrimento que se prolongam em meses ou anos. Apesar da maioria das mães engravidar após um ano, isso não significa que superaram o sofrimento anterior, essa nova gestação pode ser caracterizada por momentos de ansiedade, medo e angústia (ALEXANDRE et al., 2015), indicando a necessidade de seguimento das mães pelos serviços de saúde.

Os pais mantêm a esperança como a base para planejar a vinda de outro filho. Todavia, o medo de que a história anterior se repita fica presente durante a gestação. Muitos pais têm dificuldade de contar para as pessoas à sua volta que estão aguardando um novo filho, seja pela cobrança ou medo de reviver a história (MILLS et al., 2014).

No intuito de apoiar o processo do luto, as publicações são concordantes em citar que rituais de despedida são importantes de serem vividos pelas família, em especial as mães, porque descerram que os pais reconhecem a existência do bebê, assumindo que este existiu, teve uma vida curta, mas, foi importante para a família (STURROCK; LOUW, 2013). Porém, esse momento deve ser acompanhado pelos profissionais da saúde, proporcionando suporte e opções de escolhas sobre o ritual de despedida para a possível ressignificação da morte (CACCIATORE, 2013a).

Portanto, os profissionais devem ajudar a mãe a enfrentar as dores físicas e principalmente as psicológicas, de forma a oferecer acolhimento e escutar seus medos e anseios (LEMOS; CUNHA, 2015). Por essa razão, as pessoas que trabalham diretamente com essas mães, precisam conhecer e sentir o que querem e precisam saber, de forma que eles se sintam seguros para enfrentar o processo do luto (HUTTI, ARMSTRONG; MYERS, 2013).

Ainda, nessa revisão integrativa, foi revelado que a maioria das mães recorrem à ajuda religiosa, na convicção que Deus permitiu a morte de seus filhos e está no controle de tudo. Assim é possível confiar que com o tempo "Deus vai dar força" e ajudar a superar o momento devastador. Em contrapartida, muitas mulheres ficam entre um elo do "Por que Deus permitiu isso comigo?" e ao mesmo tempo pedem socorro para que Deus dê 
força (STURROCK; LOUW, 2013). A compreensão, pelos profissionais da saúde e familiares de que o questionamento e a aceitação fazem parte do processo do luto pode ajudar no cuidado adequado às mães mediante as etapas de luto vivenciadas. Com solidariedade, respeito ao momento e à história de cada pessoa, torna-se mais viável o aporte para que a mãe enlutada possa reconstruir uma nova realidade a partir do vazio deixado pela morte do filho.

É importante que a mãe tenha uma rede de apoio, formada pelos profissionais de saúde, família, religiosos e amigos. Entretanto, existe a necessidade de preparo para lidar com a situação gerada pelo óbito, e empatia com as pessoas que passam pela morte do bebê (LEMOS; CUNHA, 2015; LOPES et al., 2017). Em relação aos profissionais da saúde, a abordagem do tema da morte deve fazer parte de sua formação desde a graduação. Porém, não se pode negar que lidar com a morte envolve vencer os próprios medos, inseguranças, crenças, e transcender as próprias experiências para que o alvo seja apenas o cuidar do outro.

\section{Conclusão}

A revisão integrativa da literatura mostrou que as mães que vivenciam a perda de um filho no período perinatal sentem uma dor devastadora e que elas se apegam na fé para transpor este período e os sentimentos por ele provocados. Profissionais da saúde devem ser qualificados para assistência e apoio aos atores envolvidos nesse momento angustiante, favorecendo a possiblidade de rituais de despedida do bebê pela mãe e familiares, e retirando a mãe enlutada do convívio hospitalar de outras mães que tenham filhos vivos e saudáveis.

Esta revisão integrativa possui a limitação de ter envolvido um período limitado de análise da literatura (cinco anos), também englobou estudos que fazem variadas abordagens e estudam populações distintas. No entanto, optou-se pelas publicações mais recentes, as quais pudessem relatar abordagens e especificidades atuais sobre a dor de perder um bebê. Além do que, a morte perinatal, histórica e culturalmente, tem sido silenciosa pelas pesquisas e sociedade. De igual modo, como não há vastidão de estudos sobre o tema, o interesse principal era verificar todas as abordagens e tipos de pesquisas feitas sobre o tema do luto materno frente aos óbitos perinatais. Outros tipos de estudos ou revisões da literatura poderão ser realizados respondendo a objetivos de aspectos específicos da mortalidade perinatal.

Por fim, conclui-se que existem poucos estudos com evidência científica elevada sobre o luto materno e uma escassez sobre o tema na literatura brasileira. Mas, há vários estudos qualitativos que mostram as subjetividades ligadas à dor da perda de um bebê.

\section{Referências}

ALEXANDRE, $M$. et al. The impact of prior medical termination of pregnancy on the mother's early relationship with a subsequent infant. Journal of maternal-fetal \& neonatal medicine, v. 7058, p. 1-6, 2015.

ASPLIN, N. et al. Maternal emotional wellbeing over time and attachment to the fetus when a malformation is detected. Sexual and Reproductive Healthcare, v. 6, n. 3, p. 191-195, 2015.

AVELIN, P. et al. Parental grief and relationships after the loss of a stillborn baby. Midwifery, v. 29, n. 6, p. 668-673, 2013. 
BOYDEN, J. Y. et al. Experiences of african american parents following perinatal or pediatric death: a literature review. Death Studies, v. 38, p. 374-380, 2015.

CACCIATORE, J. Psychological effects of stillbirth. Seminars in Fetal and Neonatal Medicine, v. 18, n. 2, p. 76-82, 2013.

CACCIATORE, J.; ERLANDSSON, K.; RADESTAD, I. Fatherhood and suffering: A qualitative exploration of Swedish men's experiences of care after the death of a baby. International Journal of Nursing Studies, v. 50, n. 5, 2013.

CAMPBELL-JACKSON, L.; BEZANCE, J.; HORSCH, A. "A renewed sense of purpose": Mothers' and fathers' experience of having a child following a recent stillbirth. Pregnancy and Childbirth, v. 14, n. 1, p. 1-12, 2014.

CAMPOS, G. W. S.; ONOCKO-CAMPOS, T.; DEL BARRIO, L. R. Políticas e práticas em saúde mental: as evidências em questão. Ciência \& Saúde Coletiva, v.18, n.10, p.27972805, 2013.

CASELLATO, G. Luto não autorizado: o fracasso da empatia nos tempos modernos. Suporte psicológico ao luto não reconhecido. São Paulo: Summus, v.1, p. 15-28, 2015.

FLENADY, V. et al. Meeting the needs of parents after a stillbirth or neonatal death. International journal of obstetrics and gynaecology, v. 121, p. 137-140, 2014.

HEAZELL, A. et al. Sharing experiences to improve bereavement support and clinical care after stillbirth: Report of the 7th annual meeting of the international stillbirth alliance. Acta Obstetricia et Gynecologica Scandinavica, v. 92, n. 3, p. 352-361, 2013.

HILL, P. W. et al. The loss of self: The effect of miscarriage, stillbirth, and child death on maternal self-esteem. Death Studies, v. 41, n. 4, p. 226-235, 2017.

HUBERTY, J. L.; et al. A qualitative study exploring women's beliefs about physical activity after stillbirth. Pregnancy and childbirth, v. 14, n. 1, 2014.

HUTTI, M. H.; ARMSTRONG, D. S.; MYERS, J. Evaluation of the Perinatal Grief Intensity Scale in the Subsequent Pregnancy After Perinatal Loss. Journal of Obstetric, Gynecologic, and Neonatal Nursing, v. 42, n. 6, p. 697-706, 2013.

LEMOS, L. F. S.; CUNHA, A. C. B. Concepções Sobre Morte e Luto: Experiência Feminina Sobre a Perda Gestacional. Psicologia: ciência e profissão, v. 35, n. 4, p. 1120-1138, 2015.

LOPES, B. G.; et al. Luto materno: dor e enfrentamento da perda de um bebê. Revista Rene, v. 18, n. 3, p. 307-313, 2017.

MEHRAN, P. et al. History of perinatal loss and maternal-fetal attachment behaviors. Women and Birth, v. 26, n. 3, p. 185-189, 2013.

MILLS, T. A. et al. Parents' experiences and expectations of care in pregnancy after stillbirth or neonatal death: A metasynthesis. International Journal of Obstetrics and Gynaecology, v. 121, n. 8, p. 943-950, 2014. 
MURPHY, S.; JONES, K. S. By the way knowledge: Grandparents, stillbirth and neonatal death. Human Fertility, v. 17, n. 3, p. 210-213. 2014.

PARIS, G. F.; MONTIGNY, F.; PELLOSO, S. M. Adaptação transcultural e evidências de validação da perinatal grief scale. Cogitare Enfermagem, v. 26, n. 1, 2017.

PARIS, G. F.; MONTIGNY, S.; PELLOSO, S. M. Fatores associados ao estado de luto após óbito fetal: estudo comparativo entre brasileiras e canadenses. Revista da Escola de Enfermagem da USP, v. 50, n. 4, p. 546-553, 2016.

PETERS, M. D. J. et al. Caring for families experiencing stillbirth: Evidence-based guidance for maternity care providers. Women and Birth, v. 28, n. 4, p. 272-278, 2015.

PETRO, S. J. Drawing Close to the Brokenhearted: Pastoral Responses to Parents Grieving Stillbirth. The journal of pastoral care \& counseling, v. 69, n. 1, p. 13-18, 2015.

RYNINKS, K. et al. Mothers' experience of their contact with their stillborn infant: An interpretative phenomenological analysis. Pregnancy and childbirth, v. 14, n. 1, p. 203, 2014.

SOUZA, M. T.; SILVA, M. D.; CARVALHO, R. Revisão integrativa: o que é e como fazer? Einstein, v. 8, n. 1, p. 102-106, 2010.

STEEN, S. E. Perinatal death: bereavement interventions used by US and Spanish nurses and midwives. International journal of palliative nursing, v. 21, n. 2, p. 79-86, 2015.

STURROCK, C.; LOUW, J. Meaning-making after neonatal death: narratives of Xhosaspeaking women in South Africa. Death Studies, v. 37, n. 6, p. 569-588, 2013.

SUN, L. et al. Estimation of high risk pregnancy contributing to perinatal morbidity and mortality from a birth population-based regional survey in 2010 in China. Pregnancy and childbirth, v. 14, n. 1, p. 338, 2014.

USTUNDAG-BUDAK, A. M. et al. Mothers' accounts of their stillbirth experiences and of their subsequent relationships with their living infant: an interpretative phenomenological analysis. Pregnancy and childbirth, v. 15, 2015.

WOOL, C. Instrument Development: Parental Satisfaction and Quality Indicators of Perinatal Palliative Care. Journal of Hospice \& Palliative Nursing, v. 17, n. 4, p. 301 308, 2015. 